\title{
Atomization of viscous and non-newtonian liquids by a coaxial, high-speed gas jet. Experiments and droplet size modeling
}

\author{
A. Aliseda ${ }^{\text {a,1 }}$, E.J. Hopfinger ${ }^{\text {a }}$, J.C. Lasheras ${ }^{\text {a }}$, \\ D.M. Kremer ${ }^{b, *}$, A. Berchielli ${ }^{b}$, E.K. Connolly ${ }^{b}$ \\ ${ }^{a}$ Department of Mechanical and Aerospace Engineering, University of California, San Diego, 9500 Gilman Drive, \\ La Jolla, CA 92093-0411, United States \\ ${ }^{\mathrm{b}}$ Oral Products Center of Emphasis, Pfizer, Inc., Global Research and Development, Groton/New London Laboratories, \\ Eastern Point Road MS 8156-07, Groton, CT 06340, United States
}

This paper describes a collaborative theoretical and experimental research effort to investigate both the atomization dynamics of non-Newtonian liquids as well as the performance of coaxial atomizers utilized in pharmaceutical tablet coating. In pharmaceutically relevant applications, the coating solutions being atomized are typically complex, non-Newtonian fluids which may contain polymers, surfactants and large concentrations of insoluble solids in suspension. The goal of this investigation was to improve the understanding of the physical mechanism that leads to atomization of viscous and nonNewtonian fluids and to produce a validated theoretical model capable of making quantitative predictions of atomizer performance in pharmaceutical tablet coaters. The Rayleigh-Taylor model developed by Varga et al. has been extended to viscous and non-Newtonian fluids starting with the general dispersion relation obtained by Joseph et al. The theoretical model is validated using droplet diameter data collected with a Phase Doppler Particle Analyzer for six fluids of increasing rheological complexity. The primary output from the model is the Sauter Mean Diameter of the atomized droplet distribution, which is shown to compare favorably with experimental data. Critical model parameters and plans for additional research are also identified.

\footnotetext{
* Corresponding author. Tel.: +1 860686 2856; fax: +1 8606866509.

E-mail address: douglas.m.kremer@pfizer.com (D.M. Kremer).

${ }^{1}$ Current Address: Department of Mechanical Engineering, University of Washington, Stevens Way, Box 352600, Seattle, WA 98195 , United States.
} 


\section{Introduction}

The atomization of a liquid jet by a co-flowing, high-speed gas is a process of considerable practical interest in many industrial settings as well as being a fundamental research topic in multiphase flow. Although atomization processes are utilized frequently in industrial applications, the underlying physical mechanisms that determine atomization characteristics are not fully understood. In particular, while the atomization of liquids is utilized extensively in a variety of pharmaceutical manufacturing processes, a clear need remains for physicsbased models to facilitate process understanding and scale-up. The role of atomization in pharmaceutical manufacturing can be organized into two broadly defined categories. One category of pharmaceutical manufacturing processes utilizes atomization to alter the in vivo performance of the active pharmaceutical ingredient (API), often by modifying the bioavailability of the API itself. A common manufacturing process of this type is spray drying. During spray drying, API and other excipients are dissolved in solvents and the solution is atomized in a heated gas stream and dried to form powders (Masters, 1976). Research has shown that the size distribution of the atomized droplets coupled with the operating parameters of the spray dryer can influence the morphology of the dried powder (Lin and Gentry, 2003). Additionally, scale-up of the spray drying process is notoriously difficult due to the inability of models to predict atomizer performance at different scales, especially for pharmaceutically relevant solutions (Kremer and Hancock, 2006; Oakley, 2004). Thus, scale-up of this process can result in unanticipated changes in the size and morphology of the dried powder which can deleteriously impact the downstream manufacturing steps necessary to produce the final dosage form. Another example of a pharmaceutical manufacturing operation in this category is spray congealing. In this process, the API is mixed with waxes and atomized, normally via a rotary atomizer, with the goal of producing very small particles containing encapsulated API (Kawase and De, 1982; Mackaplow et al., 2006). Encapsulation can modify the release profile of the API or target dissolution of the encapsulated particle to specific regions of the gastrointestinal tract.

In the other category of applications, atomization is utilized to modify the appearance or improve the in vivo performance of the final dosage form. The most common example of this type of process is tablet coating, with a recent survey indicating that $\sim 55 \%$ of pharmaceutical tablets manufactured in 2006 were coated (IMS Midas Database, 2007). There are a number of reasons why such a large percentage of pharmaceutical tablets are coated, which adds an additional unit operation to the manufacture of the final dosage form. Nonfunctional tablet coatings improve the appearance and handling of tablets and may protect against counterfeiting by improving brand recognition. Functional tablet coatings are applied to mask unpleasant taste or alter the tablet dissolution profile either by controlling the rate of dissolution, normally via semi-permeable membrane coatings, or by protecting the tablet from the acidic environment of the stomach via enteric coatings. As is the case for spray drying, scale-up of the tablet coating process is difficult as the operation also involves several coupled physical processes occurring simultaneously. In addition to atomizing the coating solution, the tablet coating process involves mixing a bed of tablets as well as drying the coating solution on the surface of the tablets resulting in the final solid coating. Pharmaceutical researchers have developed thermodynamic models to simulate the tablet coating process and guide scale-up; however these models, while useful, make no attempt to predict atomizer performance at different scales (am Ende and Berchielli, 2005).

Atomization, and especially air-blast atomization, is a complex multi-parameter problem. For this reason, it has eluded a clear physical understanding and general theoretical predictions of the droplet size as a function of the injector geometry and fluid properties. A physical mechanism which compares satisfactorily to experimental evidence is a two-stage instability mechanism, a primary shear instability (Funada et al., 2004; Lozano et al., 2001; Yecko and Zaleski, 2005) followed by a Rayleigh-Taylor (R-T) instability of the liquid tongues produced by the primary instability (Joseph et al., 1999). In this scenario, the liquid jet diameter is practically irrelevant (Varga et al., 2003); the thickness of the gas boundary layer at the injector exit determines the wavelength of the primary instability and the subsequent fluid mass that is suddenly exposed to the gas stream and accelerated (Boeck et al., 2007; Lopez-Pages et al., 2004). For low viscosity fluids, in which viscous effects are negligible, the R-T wavelength that determines the ligament size and hence the drop size depends only on surface tension (Varga et al., 2003).

In pharmaceutically relevant applications, the liquids being atomized are typically complex, non-Newtonian fluids which may contain polymers, surfactants and high concentrations of insoluble solids in suspension. 
Tablet coating, regardless of the nature of the coating, and many pharmaceutical spray drying operations utilize coaxial air blast atomizers (Muller and Kleinebudde, 2006). Although the performance of coaxial airblast atomizers has been studied extensively (Lasheras and Hopfinger, 2000; Varga et al., 2003), very few of these investigations were focused on atomization of highly viscous or non-Newtonian liquids (Mansour and Chigier, 1995; Marmottant, 2001). In this paper, we describe a collaborative theoretical and experimental research effort to investigate the performance of commercial coaxial atomizers utilized in pharmaceutical tablet coating when atomizing common tablet coating solutions under typical processing conditions.

As such, the goal of this investigation is to produce a validated theoretical model capable of making timely predictions of atomizer performance in pharmaceutical tablet coaters. The theoretical study performed here demonstrates that for liquids with viscous or non-Newtonian properties, like many common tablet coating solutions, the R-T wavelength is strongly affected by the high viscosity or the non-Newtonian behavior of the solution. Joseph et al. (Joseph et al., 2002) demonstrated this very clearly for viscoelastic liquid drops suddenly exposed to a high-speed gas stream. In this study, the R-T model originally developed by Varga et al. (Varga et al., 2003) is extended to viscous and non-Newtonian fluids starting with the general dispersion relation developed by Joseph et al. (Joseph et al., 2002). The theoretical model is validated using data collected with Phase Doppler Particle Analysis (PDPA). The primary output from the model is the Sauter Mean Diameter of the atomized droplet distribution which is shown to compare favorably with experimental data. Critical model parameters and plans for additional research are also identified.

\section{Description of experiment}

\subsection{Experimental setup}

Experiments were carried out using a Spraying Systems atomizer (1/8 JAC series with gas cap PA11228-45C, and liquid nozzle PF28100NB) which has a well-characterized geometry shown in Fig. 1. The liquid was pressurized in a bladder tank, flowed through a calibrated flow meter and injected through a small diameter orifice at the centerline of the atomizer. Pressurized air was injected coaxially with the liquid stream through an annular gap located at the base of the liquid nozzle. Between $10 \%$ and $20 \%$ of the pressurized air flowed through auxiliary ports located in the periphery of the gas cap and oriented at a $45^{\circ}$ angle to the main liquid

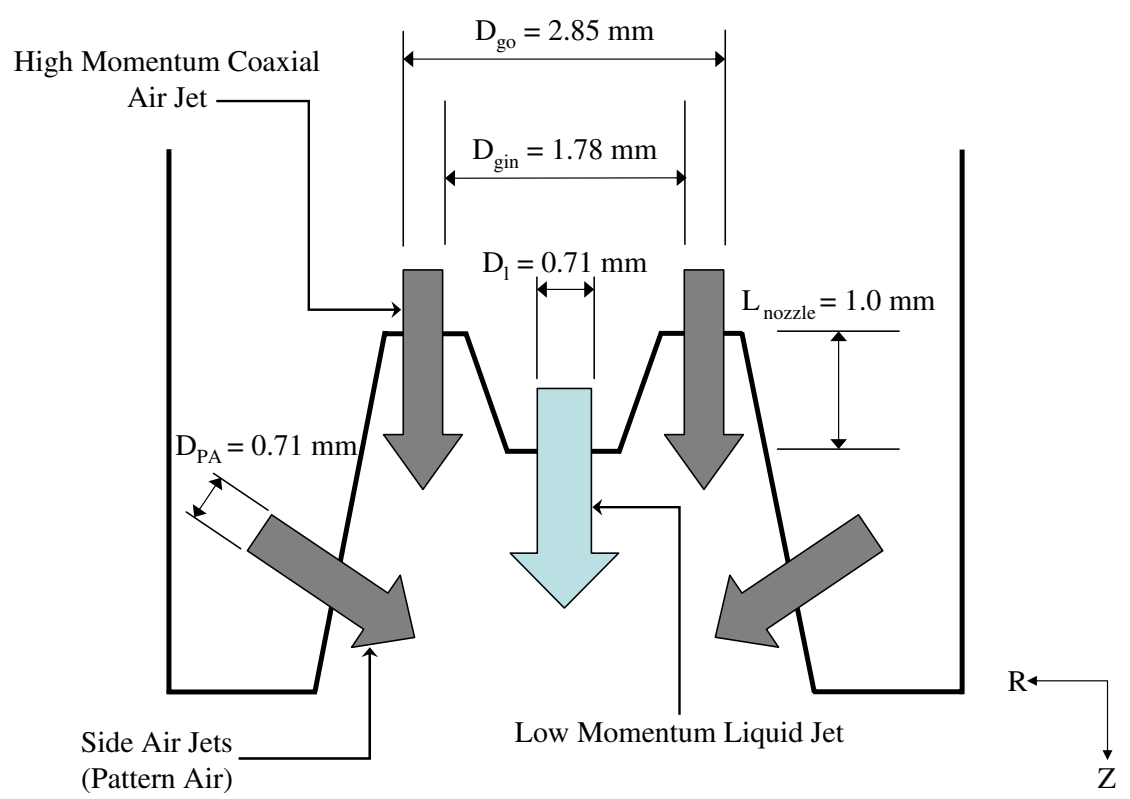

Fig. 1. Atomizer schematic. 
and gas streams (see Fig. 1 for details). This pattern air induces an asymmetry in the velocity field such that the cross section of the spray becomes elliptical. As such, the pattern air plays an important role in the transport of small liquid droplets inside the spray. However, because the pattern air merges with the main streams at a distance of more than ten liquid orifice diameters downstream of injection, it will be shown to play a negligible role in the liquid atomization process which is dominated by a series of instabilities which form very close to the liquid nozzle discharge. The air flow rate was measured by a flow meter and the outlet pressure was measured by a pressure gauge to correct for compressibility effects at the flow meter outlet. The atomizer was secured to a two-dimensional traverse system so that it could be precisely positioned with respect to the measuring point along the radial and axial coordinates of the spray. A sketch and photograph of the experimental facility is presented in Fig. 2.

\subsection{Droplet size and velocity measurements}

The velocity and size of the droplets produced during atomization were measured by a Phase Doppler Particle Analyzer (TSI Inc., Minneapolis, MN). A detailed description of this measurement technique can be found elsewhere (Bachalo, 1994). Briefly, the $514.5 \mathrm{~nm}$ beam from an Argon ion laser was split and one of the beams passed through a Bragg cell which produced a $40 \mathrm{MHz}$ frequency shift. These two beams were then transported through fiber optics to the experimental setup where they cross, forming an interferometry fringe pattern at the probe volume. Light scattered from the droplets crossing through the beams' intersection was acquired at three distinct points by the receiver and processed by three photodetectors. The frequency and phase shift in the signal were extracted to compute the droplet velocity and diameter, respectively. In these experiments, the receiver was placed at a $30^{\circ}$ angle with the transmitter to collect backscattered light and a $150 \mu \mathrm{m}$ slit was used in order to reduce the probe volume size. With the current optical setup the probe volume was $110 \mu \mathrm{m}$ in diameter and $525 \mu \mathrm{m}$ long, and the resolution of the system allows the detection of droplets down to $1.5 \mu \mathrm{m}$ in diameter.
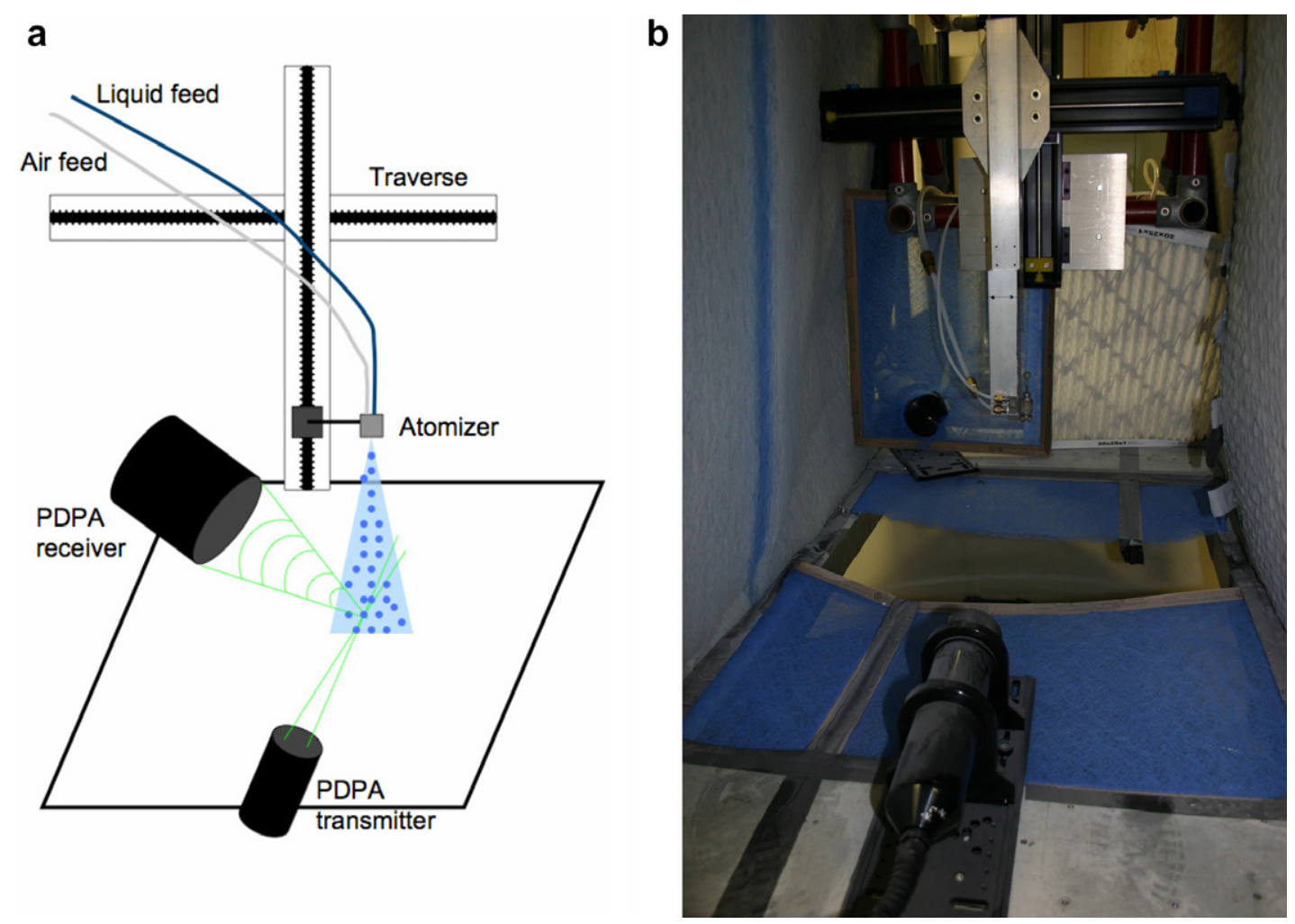

Fig. 2. (a) Sketch and (b) photograph of the atomization experiment. 
The PDPA system was positioned in such a way that the measurement volume was located on the plane where the injector nozzle evolved. The atomizer moved relative to the probe volume using the two degrees of freedom of the traverse system. Thus, measurements were taken along different radial and axial positions within a plane that cut diametrically across the spray. The origin of this plane was located at the center of the liquid nozzle discharge with the orientation of the coordinate system as indicated in Fig. 1. The axial velocity and size of individual droplets flowing through the probe volume were measured and statistically analyzed. The arithmetic mean velocity of the droplets and the Sauter mean diameter (SMD) of the droplets were computed directly from the raw measurements using MATLAB (Mathworks, Natick, MA).

High-speed visualizations of the primary break-up process were captured by back-illuminating the region of interest at the outlet of the liquid and gas jets. A Photron Fastcam $10 \mathrm{k}$ digital camera, at a resolution of $256 \times 240$ pixels, was focused through a Nikor $65 \mathrm{~mm}$ Micro lens on a $5 \mathrm{~mm} \times 5 \mathrm{~mm}$ region located at the outlet of the liquid nozzle. The camera operated at 1000 frames per second and the illumination came from a Kodak stroboscopic light synchronized with the camera. Although the exposure time of the camera was set at $1 / 2000 \mathrm{~s}$, the light pulses from the stroboscopic light were very short (approx. $10 \mu \mathrm{s}$ ) so that the droplet motion was frozen and the sharpness in the resulting images was enhanced. Images captured by this method for different experimental conditions are shown in Fig. 4.

\subsection{Characterization of liquid rheology}

Six fluids with rheologies of increasing complexity were utilized in this study, specifically water, two glycerol-water mixtures, an acetone/water/cellulose acetate (CA)/polyethylene glycol (PEG) mixture and two commercially available Opadry ${ }^{\mathrm{TM}}$ II water-based suspensions, Y-30-18037 and 85F18422 (Colorcon, West Point, PA). The CA-PEG coating was prepared by adding 9\% (w/w) CA and $1 \%$ PEG to a solution consisting of 3\% water and $87 \%$ acetone. Both Opadry ${ }^{\mathrm{mM}}$ suspensions were aqueous; however Y-30-18037 utilized 15\% solids (w/w) in suspension, composed primarily of a mixture of lactose monohydrate, hydroxypropyl methylcellulose (HPMC), titanium dioxide and triacetin, while $85 \mathrm{~F} 18422$ utilized $20 \%$ solids in solution, composed primarily of a mixture of partially-hydrolyzed polyvinyl alcohol (PVA), titanium dioxide, PEG and talc. In subsequent discussion, suspension Y-30-18037 will be referred to as Opadry ${ }^{\mathrm{TM}}$-HPMC and suspension $85 \mathrm{~F} 18422$ will be referred to as Opadry ${ }^{\mathrm{m}}-\mathrm{PVA}$.

The shear rate dependence of viscosity for the different fluids used in the atomization experiments was measured on a Brookfield DVII+Pro digital cone and plate viscometer. The viscosity of water and two different solutions of glycerol in water were tested to validate the procedure and check the viscometer calibration. The measured values were constant across all values of shear rate tested, as expected for Newtonian fluids. The rheology of the solutions of interest was also investigated within the range of shear rates available. Surface tension was measured with a Cole Parmer EW-59951 tensiometer. This system uses the du Nuoy ring method with a platinum iridium ring and a calibrated torque balance to measure the surface tension of liquids in air. The density, surface tension and viscosity at different shear rates of these fluids were measured prior to atomization and the results are given in Table 1.

The data presented in Table 1 clearly shows that the Opadry ${ }^{\mathrm{TM}}$ solutions exhibit a strong non-Newtonian behavior. The other fluids have almost constant viscosity, with variations in the different measurements attributed to slight internal heating at higher shear rates. The shear-thinning (pseudoplastic) behavior of

Table 1

Physical and rheological properties of the fluids utilized in the atomization experiments

\begin{tabular}{|c|c|c|c|c|c|c|c|}
\hline & $\begin{array}{l}\rho \\
\left(\mathrm{Kg} / \mathrm{m}^{3}\right)\end{array}$ & $\begin{array}{l}\sigma \\
(\mathrm{N} / \mathrm{m})\end{array}$ & $\begin{array}{l}\mu \times 10^{-3}(\mathrm{Kg} / \mathrm{ms}) \\
@ 30 \mathrm{~s}^{-1}\end{array}$ & $\begin{array}{l}\mu \times 10^{-3}(\mathrm{Kg} / \mathrm{ms}) \\
\text { @ } 75 \mathrm{~s}^{-1}\end{array}$ & $\begin{array}{l}\mu \times 10^{-3}(\mathrm{Kg} / \mathrm{ms}) \\
@ 150 \mathrm{~s}^{-1}\end{array}$ & $\begin{array}{l}\mu \times 10^{-3}(\mathrm{Kg} / \mathrm{ms}) \\
\text { (a) } 225 \mathrm{~s}^{-1}\end{array}$ & $\begin{array}{l}\mathrm{T} \\
\left({ }^{\circ} \mathrm{C}\right)\end{array}$ \\
\hline Water & 998 & 0.072 & 0.99 & 0.98 & 0.97 & 0.97 & 24.1 \\
\hline $59 \%$ Glycerol-water & 1150 & 0.065 & 9.42 & 9.32 & 9.18 & 9.15 & 22.5 \\
\hline $85 \%$ Glycerol-water & 1220 & 0.062 & 77.6 & 68.2 & 62.9 & 62.8 & 24.5 \\
\hline CA-PEG $10 \%$ Solids & 800 & 0.022 & 146 & 141 & 149 & 152 & 24.1 \\
\hline Opadry ${ }^{\mathrm{TM}}$-HPMC $15 \%$ Solids & 1070 & 0.040 & 192 & 160 & 139 & 133 & 24.1 \\
\hline Opadry $^{\mathrm{TM}}$-PVA $20 \%$ Solids & 1150 & 0.045 & 235 & 148 & 92 & 66 & 24.1 \\
\hline
\end{tabular}


the Opadry ${ }^{\text {TM }}$ solutions was characterized for low and intermediate values of the shear rate. The use of the highest measured shear rate viscosity in the atomization model yields a great improvement over use of the low viscosity values which would grossly overestimate the effect of viscosity on atomization. The shear rate at the outlet from the nozzle is estimated to be higher than the range tested here, thus it would be beneficial to measure the viscosity of the solutions at higher shear rates. It is also important to note the large differences in surface tension, ranging from $22 \mathrm{mN} / \mathrm{m}$ for the acetone based solution to $72 \mathrm{mN} / \mathrm{m}$ for water. This physical property has a very strong impact on atomization dynamics. If the polymer solutions, which have the highest viscosity, did not have such low surface tensions the resulting droplet size for these fluids would be orders of magnitude larger than water.

\section{Atomization model}

Varga et al. (Varga et al., 2003) demonstrated that the atomization of a liquid jet by a co-flowing, highspeed gas stream occurs via a series of instabilities. Initially, the primary Kelvin-Helmhotz instability develops in the annular shear layer present at the liquid nozzle discharge followed by a secondary Rayleigh-Taylor instability at the interface of the accelerating liquid tongues. The initial stages of this process are represented graphically in Fig. 3. The wave length of the primary instability, $\lambda_{1}$, depends on the gas boundary layer thickness, $\delta_{\mathrm{g}}$, at the gas discharge plane and is given by the following expression (Marmottant, 2001):

$$
\lambda_{1} \approx 2 \delta_{\mathrm{g}} \sqrt{\frac{\rho_{1}}{\rho_{\mathrm{g}}}}
$$

where $\rho_{1}$ and $\rho_{\mathrm{g}}$ are the liquid and gas densities, respectively. For a convergent nozzle, such as the PA11222845-C air cap used here, the gas flow at the nozzle exit is being accelerated and remains laminar such that the boundary layer thickness is

$$
\delta_{\mathrm{g}}=\frac{C b_{\mathrm{g}}}{\sqrt{R e_{\mathrm{bg}}}}
$$

where $R e_{\mathrm{bg}} \equiv U_{\mathrm{Gas}} b_{\mathrm{g}} / v_{\mathrm{Gas}}$ and the coefficient of proportionality $C$ depends on nozzle design. For the values of gas flow rate investigated here, the Reynolds number was approximately 8000 . The convective velocity of the liquid tongues resulting from this instability is

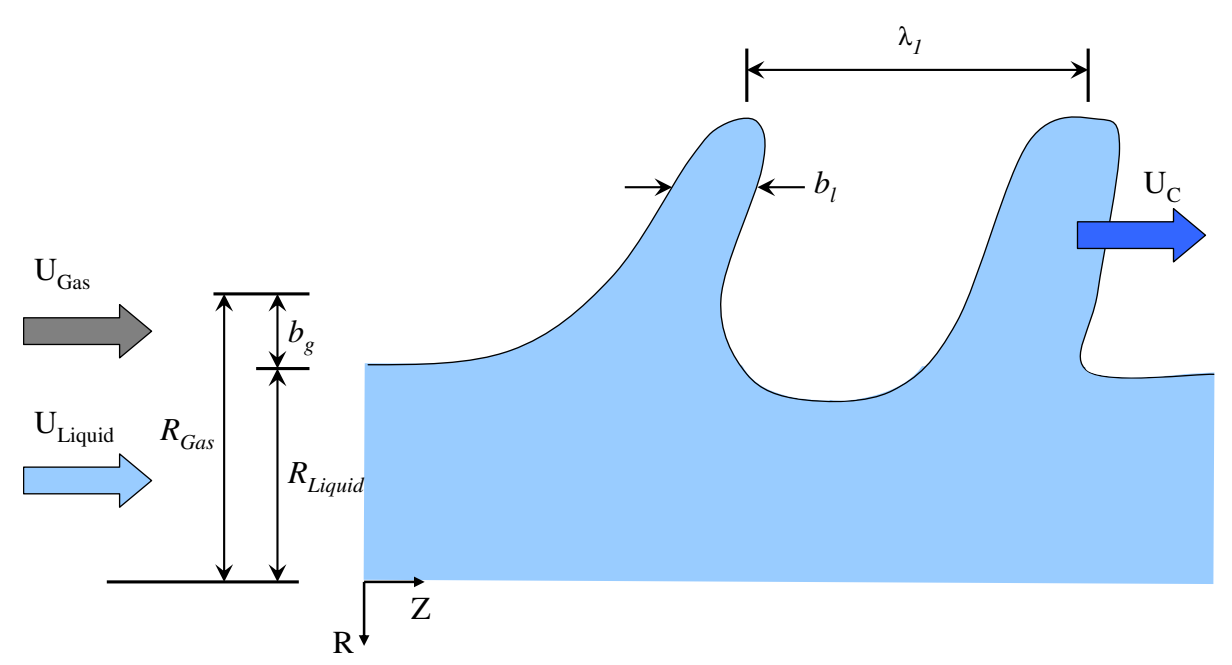

Fig. 3. Sketch of the primary instability in the liquid stream caused by the high-speed, coaxial air stream. 


$$
U_{\mathrm{c}}=\frac{\sqrt{\rho_{1}} U_{\text {Liquid }}+\sqrt{\rho_{\mathrm{g}}} U_{\text {Gas }}}{\sqrt{\rho_{1}}+\sqrt{\rho_{\mathrm{g}}}} .
$$

For the primary instability to develop rapidly it is necessary that the Reynolds number of the liquid shear layer is sufficiently large

$$
\operatorname{Re}_{\lambda 1}=\frac{\left(U_{\mathrm{c}}-U_{\text {Liquid }}\right) \lambda_{1}}{v_{1}}>10 .
$$

This condition is necessary even though the instability is driven by the gas. For non-Newtonian fluids the liquid viscosity $v_{1}$ is the effective shear viscosity which in this investigation is assumed to be the viscosity measured at the highest available shear rate, which is reasonable based on the estimated shear rate for the atomization experiments performed here.

The tongues of the primary instability, of thickness $b_{1}$, grow rapidly and are exposed to and accelerated by the high-speed gas stream. These tongues are thus subject to a R-T instability similar to a flattened drop in a high-speed gas stream. For non-Newtonian fluids the dispersion relation is given by Joseph et al. (Joseph et al., 2002) in the form (when $\rho_{\mathrm{g}} \ll \rho_{\mathrm{l}}$ )

$$
-\left[1+\frac{1}{n^{2}}\left(-a k+\frac{\sigma k^{3}}{\rho_{1}}\right)\right]+4 \frac{k^{2}}{n} \frac{\alpha_{1}}{\rho_{1}}+4 \frac{k^{3}}{n^{2}}\left(\frac{\alpha_{1}}{\rho_{1}}\right)^{2}\left(q_{1}-k\right)=0,
$$

where $k$ is the magnitude of the wave vector, $n$ the amplification rate, $a$ the acceleration of the liquid tongue, $\sigma$ the surface tension, $\alpha_{1}$ the effective shear viscosity of the liquid in $\tau_{i j}=2 \alpha_{1} e_{i j}$, where $\tau_{i j}$ and $e_{i j}$ are, respectively, the stress and rate of strain tensors in the liquid, and $q_{1}$ is given by:

$$
q_{1}=\sqrt{k^{2}+n \rho_{1} / \alpha_{1}} \text {. }
$$

When viscous effects are negligible, as in atomization of water, the wave number corresponding to maximum amplification is

$$
k_{\sigma}=\sqrt{\frac{a \rho_{1}}{3 \sigma}} .
$$

When viscous terms are important, as is the case for the water-glycerol mixtures and the tablet coating solutions under investigation here, $\alpha_{1}$ is large and it can be assumed that $\frac{n \rho_{1}}{k^{2} \alpha_{1}} \ll 1$ such that $\left(q_{1}-k\right) \approx \frac{n \rho_{1}}{2 k \alpha_{1}}$ in Eq. (6). The simplified dispersion relation from Eq. (5) then reads:

$$
n=-\frac{k^{2} \alpha_{1}}{\rho_{1}} \pm \sqrt{\frac{k^{4} \alpha_{1}^{2}}{\rho_{1}^{2}}-\left(\frac{k^{3} \sigma}{\rho_{1}}-k a\right)} .
$$

Disturbances will grow when the second term in Eq. (8) is positive and larger than the first term. It is useful to rewrite equation Eq. (8) in the form:

$$
\left.n=\frac{k^{2} \alpha_{1}}{\rho_{1}}\left[1+\frac{a \rho_{1}^{2}}{k^{3} \alpha_{1}^{2}}-\frac{\sigma \rho_{1}}{k \alpha_{1}^{2}}\right)^{1 / 2}-1\right] .
$$

From Eq. (9), the amplification rate is zero when $k=\sqrt{\frac{a \rho_{1}}{\sigma}}$, which is the capillary cut-off wave number, and when $k=0$. The wave number of maximum amplification is given by the third order equation

$$
4 \frac{\alpha_{1}^{2}}{\rho_{1}^{2}} k^{3}-\frac{3 \sigma}{\rho_{1}} k^{2}+a=0 .
$$

The exact solution of this equation is too complex to be of practical interest. However, for the high viscosity fluids studied, the Ohnesorge number (which determines the relative importance of liquid viscosity and surface tension, $O h=\frac{\alpha_{1}}{\sqrt{\rho_{1} \sigma D_{1}}}$ ) based on the wave length is large and the second term in Eq. (10) is small compared to the first one, so that the wave number of maximum amplification is: 


$$
k_{\max } \approx \sqrt[3]{\frac{a \rho_{1}^{2}}{\alpha_{1}^{2}}}
$$

The $\mathrm{R}-\mathrm{T}$ wavelength is $\lambda_{\mathrm{RT}}=\frac{2 \pi}{k_{\max }}$ and ultimately the droplet diameter is a fraction of $\lambda_{\mathrm{RT}}$ (Varga et al., 2003). Therefore, assuming viscous and surface tension effects are additive to the leading order according to the dispersion relation, we look for a correlation in the form:

$$
\lambda_{\mathrm{RT}}=2 \pi\left[\sqrt{\frac{3 \sigma}{a \rho_{1}}}+C_{2} \sqrt[3]{\frac{\alpha_{1}^{2}}{a \rho_{1}^{2}}}\right] .
$$

The acceleration $a$ in Eq. (12) is simply $a=\frac{F}{m}=\frac{F}{\rho_{1} V}$, where the force $F$ is the drag force exerted by the gas stream on a liquid element, here the liquid tongue of the primary instability,

$$
F=\frac{1}{2} C_{\mathrm{D}} \rho_{\mathrm{g}}\left(U_{\mathrm{Gas}}-U_{\mathrm{c}}\right)^{2} A_{\mathrm{e}}
$$

where $C_{\mathrm{D}} \approx 2$ is the drag coefficient and $A_{\mathrm{e}}$ the projected area. The mass of the liquid to be accelerated is $m=\rho_{1} b_{1} A_{\mathrm{e}}$ with $b_{1} \propto \lambda_{1}$. The expression for $a$ is therefore given by:

$$
a \approx \frac{\rho_{\mathrm{g}}\left(U_{\mathrm{Gas}}-U_{\mathrm{c}}\right)^{2}}{\rho_{\mathrm{l}} b_{1}} .
$$

Substitution of Eq. (14) in Eq. (12) gives:

$$
\left.\lambda_{\mathrm{RT}} \propto \frac{\sigma \lambda_{1}}{\rho_{\mathrm{g}}\left(U_{\mathrm{g}}-U_{\mathrm{c}}\right)^{2}}\right)^{1 / 2}\left(1+C_{2}^{\prime}\left\{\frac{\rho_{\mathrm{g}}\left(U_{\mathrm{g}}-U_{\mathrm{c}}\right)^{2}}{\lambda_{1} \sigma}\right\}^{1 / 6}\left\{\frac{\alpha_{1}^{2}}{\rho_{1} \sigma}\right\}^{1 / 3}\right) .
$$

Further substituting for $\lambda_{1}$ from Eq. (1), using Eq. (2) and taking the drop diameter, say the Sauter Mean Diameter (SMD), proportional to $\lambda_{R T}$ gives:

$$
\frac{\mathrm{SMD}}{D_{\mathrm{l}}}=C_{1}\left(1+m_{\mathrm{r}}\right)\left(\frac{b_{\mathrm{g}}}{D_{\mathrm{l}}}\right)^{1 / 2}\left(\frac{\rho_{\mathrm{l}} / \rho_{\mathrm{g}}}{R e_{\mathrm{bg}}}\right)^{1 / 4} \frac{1}{\sqrt{W e_{\mathrm{Dl}}}}\left\{1+C_{2}\left(\frac{D_{\mathrm{l}}}{b_{\mathrm{g}}}\right)^{1 / 6}\left(\frac{R e_{\mathrm{bg}}}{\rho_{1} / \rho_{\mathrm{g}}}\right)^{1 / 12} W e_{\mathrm{Dl}}^{1 / 6} O h^{2 / 3}\right\} .
$$

In Eq. (16), the mass loading effect in the form $\left(1+m_{\mathrm{r}}\right)$ is obtained from energy arguments previously outlined by Mansour and Chigier (Mansour and Chigier, 1995), where $m_{\mathrm{r}}=\frac{m_{1}}{m_{\mathrm{g}}}=\frac{\rho_{\mathrm{l}} U_{\mathrm{Liquid}} A_{1}}{\rho_{\mathrm{g}} U_{\mathrm{Gas}} A_{\mathrm{g}}}$ and $A_{1}$ and $A_{\mathrm{g}}$ are the areas of the liquid and gas nozzle exit sections, respectively. Furthermore, this equation indicates a dependency of the SMD on $U_{\mathrm{Gas}}^{-5 / 4}$ and $\sigma^{-1 / 2}$. The drop diameter increases with $b_{\mathrm{g}}^{1 / 4}$ if the coefficient of proportionality $C$ in Eq. (3) remains constant when $b_{\mathrm{g}}$ is changed. As will be shown below, this would only be the case if the length of the gas jet potential cone is much larger than the liquid jet's intact length which is not typical of pharmaceutical atomizer designs.

The SMD in Eq. (16) has been made dimensionless by the liquid orifice diameter $D_{1}$ and the Weber and Ohnesorge numbers are based on $D_{1}$ following the usual convention. However, it should be emphasized that the drop diameter does not depend on the liquid orifice diameter but rather on the gas boundary layer thickness at the nozzle exit. This has been clearly demonstrated by Varga et al. (Varga et al., 2003) where the liquid orifice diameter was changed by a factor of 3 and the drop diameter remained practically identical for the same gas flow conditions. 
For completeness, the various non-dimensional parameters in Eq. (16) are defined as follows:

$$
\begin{aligned}
& \text { Weber number : } W e_{\mathrm{Dl}}=\frac{\rho_{\mathrm{g}}\left(U_{\mathrm{Gas}}-U_{\mathrm{c}}\right)^{2} D_{1}}{\sigma}, \\
& \text { Ohnesorge number }: O h=\frac{\alpha_{1}}{\sqrt{\rho_{\mathrm{l}} \sigma D_{1}}}, \\
& \text { Reynolds number }: \operatorname{Re} e_{\mathrm{gg}}=\frac{U_{\mathrm{Gas}} b_{\mathrm{g}}}{v_{\mathrm{g}}}, \\
& \text { Mass flux ratio : } m_{\mathrm{r}}=\frac{\rho_{\mathrm{l}} U_{\mathrm{Liquid}} A_{1}}{\rho_{\mathrm{g}} U_{\mathrm{Gas}} A_{\mathrm{g}}} .
\end{aligned}
$$

The coefficients $C_{1}$ and $C_{2}$ in Eq. (16) are order 1 and values for both coefficients are determined from experiments. The value of $C_{1}$ depends on the gas nozzle geometry in general, and on the contraction ratio in particular, because for a given nozzle size the gas boundary layer thickness at the liquid nozzle discharge depends strongly on the contraction ratio. $C_{2}$ characterizes the viscosity dependence of the critical wavenumber in the $\mathrm{R}-\mathrm{T}$ instability, compared to the surface tension dependence. This value is associated to the additivity and linearity of both cohesive effects, surface tension and viscosity, which determine the growth rate of the instability. The validity of the linear theory for $\mathrm{R}-\mathrm{T}$ instability has been confirmed for a wide parameter range via qualitative observation of the jet break-up process.

Another important parameter, which does not appear explicitly in Eq. (16), is the dynamic pressure ratio $M$ that determines the rate of atomization and hence the intact length of the liquid stream (Lasheras and Hopfinger, 2000). This ratio is defined as

$$
M=\frac{\rho_{\mathrm{g}} U_{\text {Gas }}^{2}}{\rho_{1} U_{\text {Liquid }}^{2}} .
$$

The dimensionless intact length of the liquid stream can be defined as $\frac{L}{D_{1}} \approx \frac{6}{\sqrt{M}}$ and in the present investigation $M$ is typically large (of the order 100). The gas potential cone length is approximately $6 b_{\mathrm{g}}$. For efficient atomization it is desirable that the gas potential cone length be equal to or larger than the liquid intact length so that the primary atomization is completed before the gas velocity starts to decrease. This requirement is expressed by

$$
\frac{b_{\mathrm{g}} \sqrt{M}}{D_{1}}>1 \text {. }
$$

It is worth noting that for the flow rates and atomizer utilized in this investigation Eq. (19) is satisfied easily, with values typically exceeding 10 , strongly suggesting that atomization in pharmaceutical tablet coating is typically quite rapid and efficient. Finally, the fluid jets under the conditions of interest here are laminar but would potentially become turbulent if the flow rates are significantly increased. Turbulent conditions in the liquid stream at the nozzle discharge plane would have little effect on the atomization process, while turbulent conditions in the high-speed gas stream would require altering the exponent of $R e_{\mathrm{bg}}$ in Eq. (16).

\section{Rheological properties}

The non-Newtonian behavior in Eq. (5) is expressed by the effective viscosity, $\alpha_{1}$ relating the stress tensor with rate of strain tensor

$$
\tau_{\mathrm{ij}}=2 \alpha_{1} e_{\mathrm{ij}} .
$$

Mansour \& Chigier (Mansour and Chigier, 1995) considered air-blast atomization of power law liquids with the shear viscosity of the form:

$$
\alpha_{\mathrm{ls}}=\mu_{1} \dot{\gamma}^{m-1} \text {. }
$$

In Eq. (21), the subscript $s$ is added to distinguish shear dependent viscosity from elongation strain dependent viscosity. Although elongational strain is dominant within the liquid nozzle (Mansour and Chigier, 1995), during atomization shear is anticipated to be much larger than elongational strain. When $m=1$ in Eq. (21) the 
shear viscosity is just the liquid viscosity, for $m<1$ the liquid is shear thinning and for $m>1$ it is shear thickening. An estimate of the shear rate in the atomization process is given by $U_{c} / \lambda_{1}$ such that $\alpha_{1}$ in (16) may be replaced by $B \mu_{1}\left(U_{\mathrm{c}} / \lambda_{1}\right)^{m-1}$ where $B$ is a constant to be determined from experiments.

In the simplest rheological model of the fluids used, we can assume the Non-Newtonian behavior will manifest in three different stages according to the value of the local shear rate experienced by the fluid. For very low shear rates, the evolution of viscosity with the rate of shear strain can be modelled by an inverted parabola $\alpha=\mu_{0}\left[1-\left(\frac{\gamma}{\gamma_{0}}\right)\right]^{2}$. For an intermediate range of shear rates, the behavior of the fluid viscosity with varying values of the rate of shear strain is captured by the classical power law $\alpha=\mu_{1}\left(\frac{\gamma}{\gamma_{1}}\right)^{m-1}$. Finally, for the larger values of the shear rate, viscosity reaches an asymptotic value that can be determined at values of the rate of shear strain for which the power law dependency is no longer valid. Typically this asymptotic behavior determines the effective viscosity for the break-up process $\mu_{\infty}$, as the fluid being atomized is subjected to very large shear deformations (Mansour and Chigier, 1995). Thus, the value of viscosity obtained at the largest shear rate is utilized for the model. This value represents a conservative estimate of the shear during atomization but avoids extrapolation based on the constitutive power law equation outside the range of shear rates tested. Despite this simplifying assumption the model data will be shown to compare favorably with experimental data.

\section{Results and discussion}

\subsection{Qualitative observations}

Images of liquid jet break-up extracted from high-speed visualizations are shown in Fig. 4 for several Weber and Reynolds numbers for both water and the $85 \%$ glycerol-water solutions. At the lower Weber numbers (which correspond to lower values of the gas Reynolds number) the primary Kelvin-Helmholtz instability grows slowly and several intact wavelengths are observed prior to break-up. At larger Weber numbers, the primary instability grows to a large amplitude more quickly, generally within one or two wavelengths, and in these cases the secondary R-T instability can be clearly observed. As such, the liquid mass at the peak of the large amplitude primary instability is exposed to the high-speed gas stream and, due to aerodynamic drag, is subject to a sudden acceleration perpendicular to the orientation of the interface. Acceleration of the interface results in the $\mathrm{R}-\mathrm{T}$ instability creating ligaments of fluid which eventually break-up into droplets. The entire break-up process can be observed clearly in Fig. $4 \mathrm{~b}$ for water (left column). The coefficient $C_{2}$ in Eq. (16) and the effect of viscosity on the atomization process can be elucidated from observation of the glycerolwater mixtures. For example, in comparing Fig. $4 \mathrm{a}$ and $\mathrm{b}$ the effect of viscosity is apparent and acts to hinder the growth of both instabilities resulting in longer break-up times and larger droplets (all other parameters being equal). This qualitative tendency is captured well by the model, as will be shown in the following subsection. The value of the coefficient $C_{2}$ is found by estimating the wavelength of the R-T instability using image processing. For the range of parameters and atomizer geometry studied a value of $C_{2}$ equal to 1 was found to agree favorably with experimental data.

The geometry of the atomizer used in this investigation was such that the pattern air impinges on the main streams at a distance downstream which is large compared to the formation of instabilities leading to droplet formation. As was discussed previously, the growth of the R-T instability whose wavelength ultimately determines the droplet size is assumed to take place in the potential cone of the main gas jet, a condition which is necessary for efficient atomization. For the conditions under investigation here this distance is on the order of $3 \mathrm{~mm}$. The distance at which the pattern air impinges on the main flow streams is $\sim 7 \mathrm{~mm}$ for the atomizer used in this investigation. For visual reference, the axial extent of the images shown in Fig. 4 is $5 \mathrm{~mm}$, thus the pattern air impinges downstream of the field of view shown. Therefore, the effect of pattern air can be neglected in the atomization model.

\subsection{Droplet size measurements and comparison with model prediction}

Predictions from the quantitative model were compared with experimental results collected on the centerline of the nozzle for the six fluids discussed previously. Conditions for the atomization experiments were 

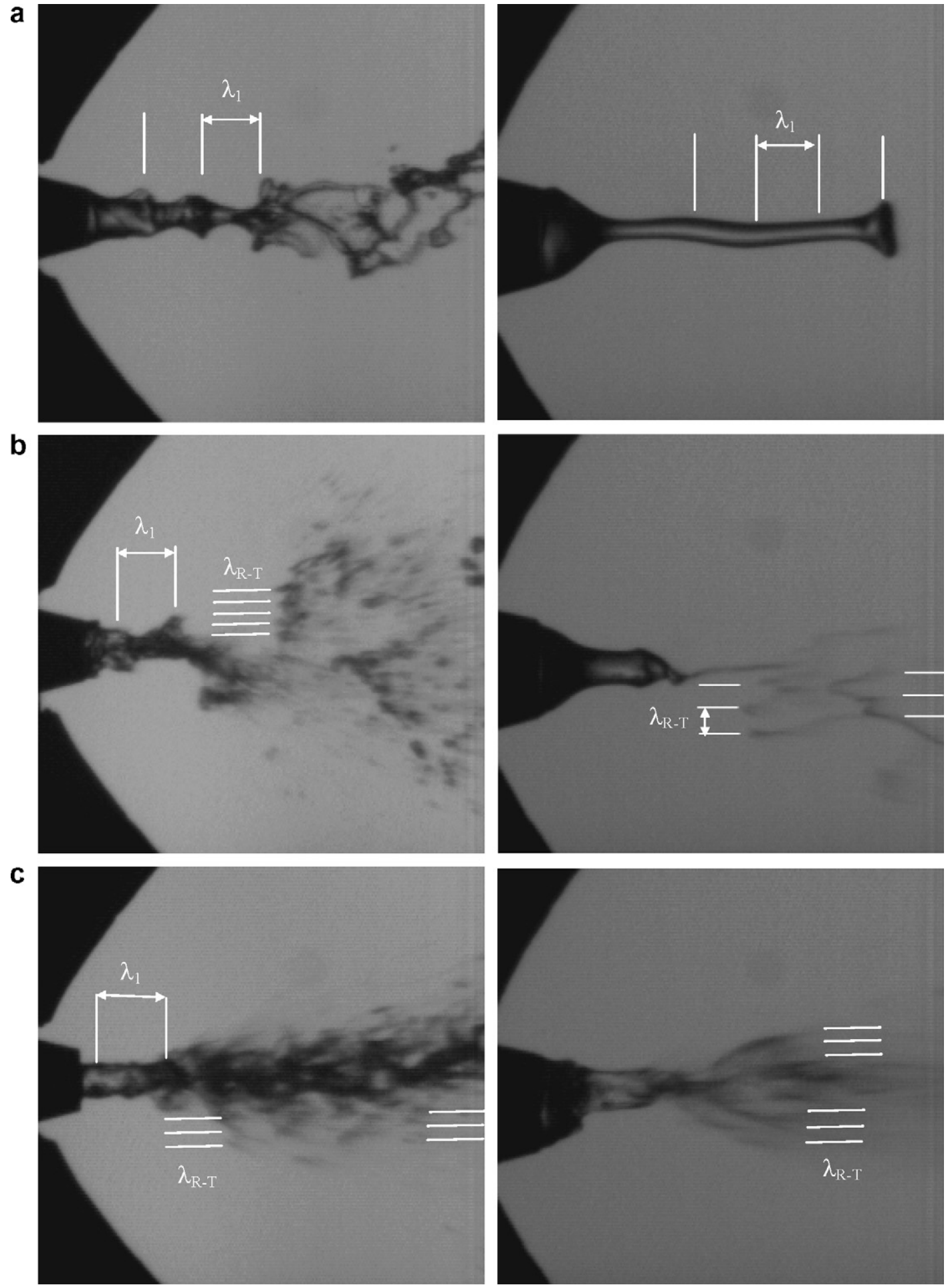

Fig. 4. High-speed visualizations of the atomization process for water (left) and $85 \%$ glycerol-water (right) at various atomization conditions. (a) $W e_{\mathrm{Dl}}=60$; (b) $W e_{\mathrm{DI}}=153$; (c) $W e_{\mathrm{Dl}}=640$.

invariant over the range of fluids tested and were conducted using a liquid flow rate of $10 \mathrm{~g} / \mathrm{min}$ and a gas flow rate of 59.5 SLPM. For the atomizer and flow rates utilized in this investigation this results in a gas velocity in 
the annular gap at the base of the liquid nozzle of $\sim 220 \mathrm{~m} / \mathrm{s}$ and the velocity of the liquid stream was approximately $0.4 \mathrm{~m} / \mathrm{s}$. The dynamic pressure ratio $(M)$ for water was $\sim 317$ which was a typical value over the range of fluids under the conditions studied here. The data from water and the two glycerol-water mixtures, shown in Fig. 5, was used to validate the model and to determine if appropriate values were assigned to the two adjustable constants in the model. As Fig. 5 demonstrates, setting both constants equal to 1 produces satisfactory model predictions for conditions of efficient atomization and a laminar gas-stream boundary layer. The values of droplet SMD predicted by the model are $19 \mu \mathrm{m}$ for water, $24 \mu \mathrm{m}$ for $59 \%$ glycerol-water and $43 \mu \mathrm{m}$ for $85 \%$ glycerol-water.

The experimental data reveals the complexity of the spray dynamics, in which the droplet size distribution evolves in a non-monotonic manner as different physical mechanisms become important. The liquid stream is first broken into large, non-spherical parcels that affect the quantitative accuracy of the PDPA measurement of SMD. The subsequent evolution of droplet sizes is consistent with the continuous break-up of ligaments resulting from the $\mathrm{R}-\mathrm{T}$ instability. At a certain distance downstream from the atomizer the droplet size distribution reaches a minimum and the break-up process is complete. It is important to emphasise that the model is predicting the SMD of the droplet distribution at the completion of atomization, which for these conditions generally occurs at an axial distance of $\sim 50 \mathrm{~mm}$ downstream from the atomizer. In pharmaceutical tablet coating the distance from the atomizer to the tablet bed is generally greater than $100 \mathrm{~mm}$ and does vary somewhat depending on the scale and manufacturer of the equipment.

After atomization is complete, as indicated by the SMD minima, the droplet size increases slowly with increasing axial distance from the atomizer. Three physical mechanisms are responsible for the observed growth in droplet size. Differential turbulent diffusion causes small droplets to travel away from the center of the spray. Coalescence, although almost negligible for these low mass loading sprays, results in larger droplets which affect the statistical values of the droplet distribution. Finally, evaporation affects small droplets causing them to decrease in size and fall below the detection threshold of the instrument. These effects cause statistical descriptions of the droplet size distribution, such as the SMD, to grow slightly. Flow visualizations in the downstream regions of the spray, which can determine the relative importance of these mechanisms quantitatively, were outside the scope of this investigation; however the three phenomena have been described

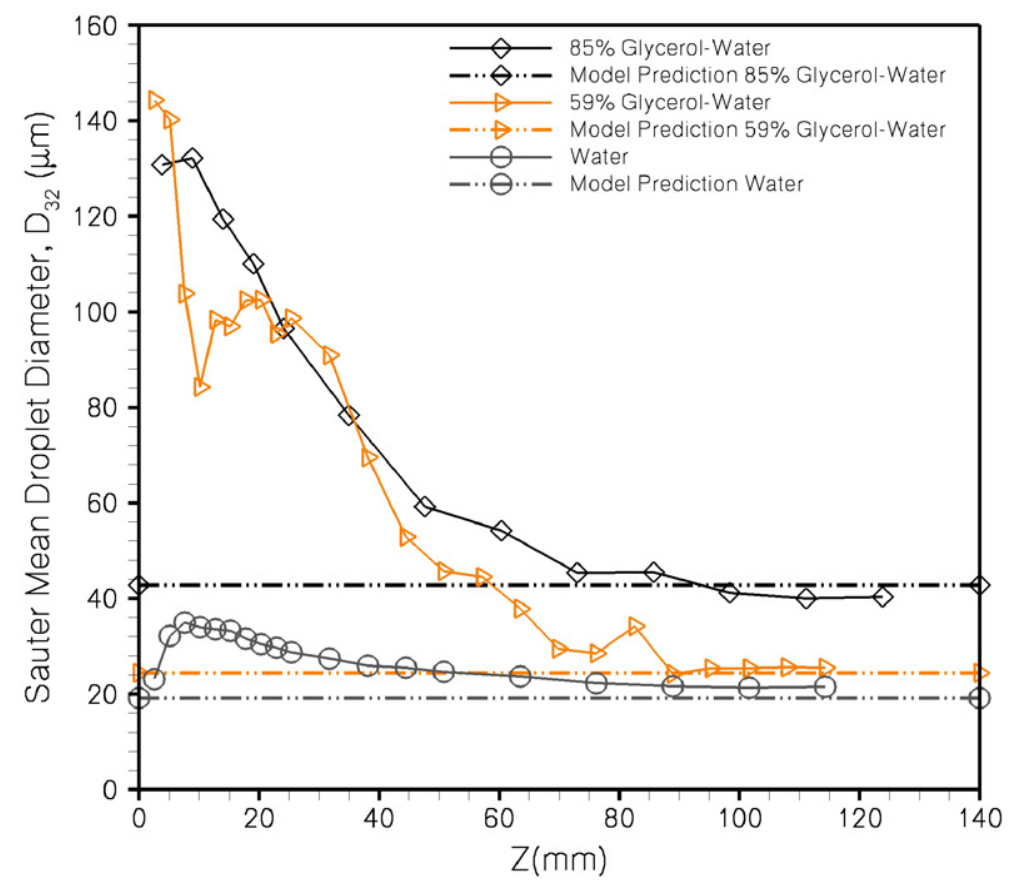

Fig. 5. Sauter Mean Diameter of water and the two glycerol-water mixtures downstream of the atomizer with corresponding model predictions. 
in descending order of estimated importance. Unlike evaporation, whose effect is larger for distributions of very small droplets, coalescence and differential turbulent diffusion are more significant for larger droplets. Thus a more significant growth in SMD is anticipated for distributions composed of larger droplets.

The kinematic viscosity of the 59\% and $85 \%$ glycerol-water mixtures are approximately 10 and 65 times that of water. It can be seen in Fig. 5 that the droplet SMD is not affected when the kinematic viscosity is ten times that of water; however the droplet SMD becomes noticeably larger when the viscosity is of the order of sixty-five times that of water. This behavior is well represented by the additive terms in Eq. (15). When viscosity is low, the first term, which depends solely on surface tension, is dominant and the dependence on viscosity is negligible. When viscosity is high, however, the term multiplying $C_{2}$ is much larger and the dependency on surface tension becomes weaker, resulting in a dominant contribution from the viscous term. Additionally, increasing viscosity also increases the distance at which atomization is complete. This is due to the fact that both the primary and secondary instabilities grow more slowly when the viscosity is large. Variation of the droplet SMD with increasing effective viscosity was found to be well captured by the additive dependency of the Ohnesorge and the Weber numbers incorporated within the model.

Data for the three tablet coating solutions is presented in Fig. 6. The values of SMD predicted by the model were $59 \mu \mathrm{m}$ for CA-PEG, $57 \mu \mathrm{m}$ for Opadry ${ }^{\mathrm{m}}-\mathrm{HPMC}$ and $41 \mu \mathrm{m}$ for Opadry ${ }^{\mathrm{m}}-\mathrm{PVA}$. These values compared favorably with the experimental values measured downstream from the injector after atomization is complete. The data presented in Figs. 5 and 6 represents the entire droplet size distribution with a single statistical value. This allows the model to predict the behavior of the droplet distribution resulting from the atomization process. Hence, the model and analysis of the data relies on the assumption that the droplet distribution can be described by a single parameter, in this case one of the moments of the distribution. In this investigation the results justify this assumption, as the measured droplet distributions were well represented by a lognormal distribution. Unlike other distribution functions, the lognormal distribution function is fully determined by a single moment. Significantly different operating conditions or injector designs may lead to other types of droplet distributions, and thus may require two or more statistical moments to be fully determined.

The data and model predictions shown here represent an initial validation of the model described in Section 3. Although a number of simplifying assumptions have been employed, this model attempts, for the first time, to capture the effect of viscosity in the break up process of highly viscous and non-Newtonian liquids and to

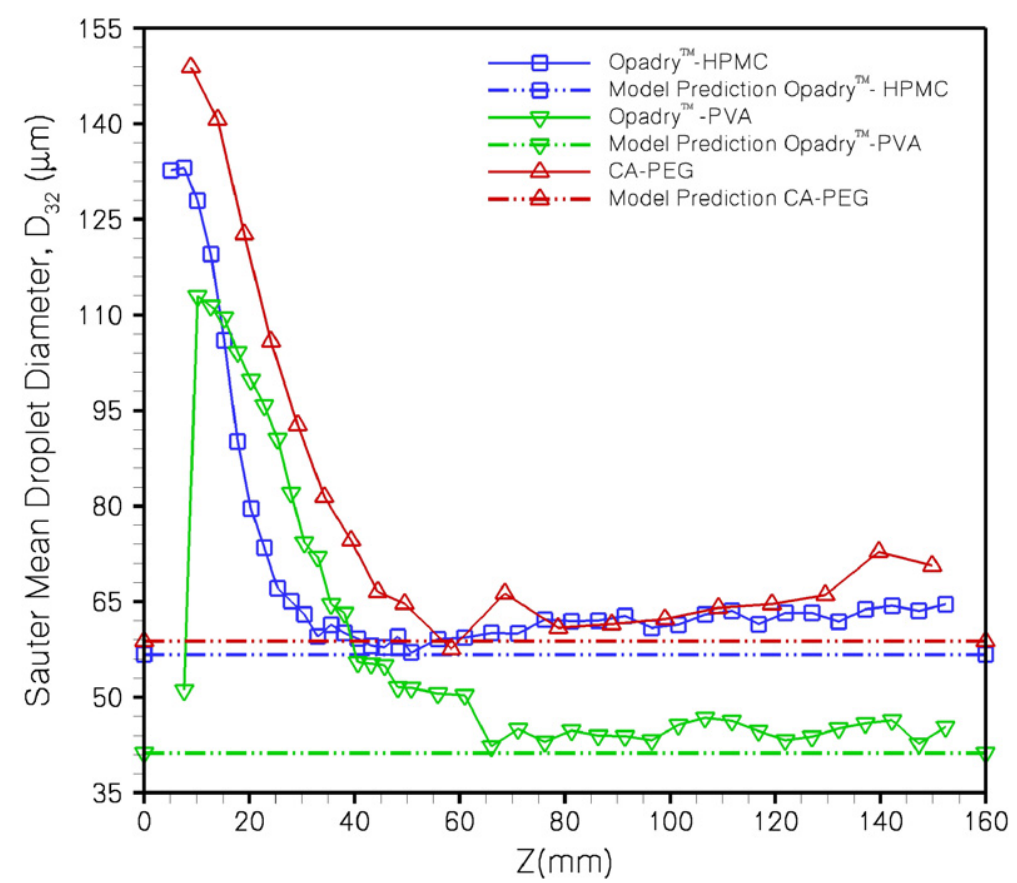

Fig. 6. Sauter Mean Diameter of three tablet coating solutions downstream of the atomizer with corresponding model predictions. 
quantitatively predict the resulting droplet sizes. The model described here is consistent with the work of Varga et al. (Varga et al., 2003) in that a stability analysis is used to predict characteristic droplet size from the most unstable wavelength of the R-T instability. Unlike the work of Mansour and Chigier (Mansour and Chigier, 1995) and Lefebvre (Lefebvre, 1996), the model does not use empirical correlations to obtain the parametric dependence of the dominant relevant non-dimensional groups (Reynolds, Weber and Ohnesorge numbers) but rather the dependency arises from first principles.

\section{Conclusions}

This work presents a model developed to predict the performance of coaxial atomizers utilized in pharmaceutical tablet coating when atomizing common tablet coating solutions under typical processing conditions. This model has been validated using fluids of increasing rheological complexity. Output from the model is the SMD of the atomized droplets after completion of atomization. The model results were found to compare favorably with experimental data over the range of fluids tested. In addition, development of the model has yielded useful insights into the characteristics and performance of atomizers frequently encountered in pharmaceutical tablet coating. For example, this investigation has clearly demonstrated that, for typical processing conditions, pattern air plays a negligible role in the atomization process which occurs via a series of instabilities which form very close to the atomizer discharge plane.

This work has also identified areas which require further investigation. Several of the fluids under investigation here are non-Newtonian and the range of shear rates at which viscosity was measured is below what would be encountered during atomization. Clearly, characterization of the rheological properties of the fluids at conditions which more closely approximate the atomization process would be expected to improve the accuracy of this model. However, the results presented here suggest that the current model is capable of making timely predictions of atomizer performance in pharmaceutical tablet coaters and offers a practical tool to guide scale-up and optimization in these systems.

\section{Acknowledgements}

Helpful discussions concerning tablet coating were held with several Pfizer colleagues, specifically A. G. Thombre, B. A. Johnson and P. E. Luner. The support of this research by the management of Pfizer Global Research and Development (S. M. Herbig, C. A. Oksanen and C. M. Sinko) is gratefully acknowledged. Finally, J. Rodriguez at UCSD offered valuable assistance with several aspects of this project.

\section{References}

am Ende, M.T., Berchielli, A., 2005. A thermodynamic model for organic and aqueous tablet film coating. Pharm. Dev. Technol. 10, 4758.

Bachalo, W.D., 1994. Experimental methods in multiphase flows. Int. J. Multiphase Flow 20, 261-295.

Boeck, T., Li, J., Lopez-Pages, E., Yecko, P., Zaleski, S., 2007. Ligament formation in sheared liquid-gas layers. Theor. Comput. Fluid Dyn. 21, 59-76.

Funada, T., Joseph, D.D., Yamashita, S., 2004. Stability of a liquid jet into incompressible gases and liquids. Int. J. Multiphase Flow 30, 1279-1310.

IMS Midas Database, 2007. IMS Health, CT, USA.

Joseph, D.D., Belanger, J., Beavers, G.S., 1999. Breakup of a liquid drop suddenly exposed to a high-speed gas stream. Int. J. Multiphase Flow 25, 1263-1303.

Joseph, D.D., Beavers, G.S., Funada, T., 2002. Rayleigh-Taylor instability of viscoelastic drops at high Weber numbers. J. Fluid Mech. 453, 109-132.

Kawase, Y., De, A., 1982. Ligament-type disintegration of non-Newtonian fluid in spinning disk atomization. J. Non-Newtonian Fluid Mech. 10, 367-371.

Kremer, D.M., Hancock, B.C., 2006. Process simulation in the pharmaceutical industry: a review of some basic physical models. J. Pharm. Sci. 95, 517-529.

Lasheras, J.C., Hopfinger, E.J., 2000. Liquid jet instability and atomization in a coaxial gas stream. Annu. Rev. Fluid Mech. 32, $275-308$. Lefebvre, A.H., 1996. Some recent developments in twin-fluid atomization. Part. Part. Syst. Char. 13, 205-216.

Lin, J.-C., Gentry, J.W., 2003. Spray drying drop morphology: experimental study. Aerosol Sci. Technol. 37, 15-32. 
Lopez-Pages, E., Dopazo, C., Fueyo, N., 2004. Very-near-field dynamics in the injection of two-dimensional gas jets and thin liquid sheets between two parallel high-speed gas streams. J. Fluid Mech. 515, 1-31.

Lozano, A., Barreras, F., Hauke, G., Dopazo, C., 2001. Longitudinal instabilities in an air-blasted liquid sheet. J. Fluid Mech. 437, 143173.

Mackaplow, M.B., Zarraga, I.E., Morris, J.F., 2006. Rotary spray congealing of a suspension: effect of disk speed and dispersed particle properties. J. Microencapsul. 23, 793-809.

Mansour, A., Chigier, N., 1995. Air-blast atomization of non-Newtonian liquids. J. Non-Newtonian Fluid Mech. 58, 161-194.

Marmottant, P., 2001. Atomisation d'un courant liquide dans un courant gazeux, Ph.D. Thesis, Institut National Polytechnique de Grenoble, Grenoble.

Masters, K., 1976. Spray Drying: An Introduction to Principles, Operational Practice and Applications. Wiley, New York.

Muller, R., Kleinebudde, P., 2006. Comparison study of laboratory and production spray guns in film coating: effect of pattern air and nozzle diameter. Pharm. Dev. Technol. 11, 425-435.

Oakley, D.E., 2004. Spray dryer modeling in theory and practice. Drying Technol. 22, 1371-1402.

Varga, C.M., Lasheras, J.C., Hopfinger, E.J., 2003. Initial breakup of a small-diameter liquid jet by a high speed gas stream. J. Fluid Mech. 497, 405-434.

Yecko, P., Zaleski, S., 2005. Transient growth in two-phase mixing layers. J. Fluid Mech. 528, 43-52. 